\title{
Imprinting in older ducklings: Some tests of a reinforcement model
}

\author{
STEPHEN J. GAIONI, HOWARD S. HOFFMAN, and PETER DePAULO \\ Bryn Mawr College, Bryn Mawr, Pennsylvania 19010
}

and

\author{
VALERIE NEWBY STRATTON \\ Pennsylvania State University, University Park, Pennsylvania 16802
}

\begin{abstract}
Ducklings (Anas Platyrhynchos domesticus) older than the so-called critical period (Days 1 and 2 posthatch) were exposed to an imprinting stimulus after various experimental histories. The first study found that in previously isolated 10-day-old subjects the stimulus exhibited the same capacity to reinforce an operant response and to generate a burst-like pattern of responding as in ducklings imprinted to it on Day 1. In Experiment 2, an imprinting stimulas exhibited reinforcing capacities in 5 to 10 -day-old ducklings that had previously been imprinted to a different stimulus. Most of these ducklings came to prefer the second imprinting stimulus over the first. Experiment 3 revealed that in 5-day-old ducklings with a prior history of imprinting to a different stimulus, the otherwise neutral features of a new imprinting stimulus acquired the same kind of persistent control over distress vocalization that they acquire in younger, naive subjects. In all these studies, the only difference between imprinting in older vs. younger subjects was that in older subjects a novel imprinting stimulus initially evoked fear reactions rather than filial behavior. These findings contradict the traditional view of imprinting as an irreversible process that occurs only during a brief critical period, but are entirely consistent with a reinforcement model of imprinting.
\end{abstract}

The traditional view of imprinting (Eibl-Eibesfeldt, 1975; Hess, 1959, 1973; Lorenz, 1935) holds that the process is distinct from learning, that its occurrence is restricted to a brief critical period early in a subject's life, and that its effects are largely irreversible. Indeed, the very term "imprinting" implies that it is a process through which an image of a stimulus is somehow indelibly stamped on a subject's nervous system, and that once this has occurred, subsequent imprinting is precluded. Recently, however, Hoffman and Ratner (1973) have formulated a reinforcement model of imprinting, which holds that the phenomenon is a form of classical conditioning and which suggests that imprinting is far less constrained by factors of age and experience than is implied by the traditional view.

According to a reinforcement model, any attachment figure-either the organism's own mother or some other potentially effective imprinting objectcan be viewed as a complex stimulus that displays

This research was supported by National Institute of Mental Health Grant MH 19715 directed by Howard S. Hoffman. Dr. Gaioni is now at the University of North Dakota, Grand Forks, North Dakota. Dr. DePaulo is now at St. Joseph's College, Philadelphia, Pennsylvania: Requests for reprints should be sent to Dr. Howard S. Hoffman, Department of Psychology, Bryn Mawr College, Bryn Mawr, Pennsylvania. a number of different features (e.g., it has a particular color, shape, size, sound, movement, etc.). The reinforcement model postulates that some of these features innately elicit filial behavior and serve as primary reinforcers, while others are initially neutral in that they do not innately evoke filial behavior and they do not reinforce ongoing responses. According to the model, when a young organism (for example, a newly hatched duckling) is exposed to an appropriate imprinting object, the innately reinforcing features of the object serve as unconditioned stimuli and are "paired" in space and time with the object's initially neutral features. As a result of this pairing, the initially neutral features of the object gradually come to elicit filial reactions as conditioned responses.

The model also postulates that as an organism matures, appropriate imprinting objects continue to be sources of reinforcing stimulation, and the system(s) responsible for the classical conditioning process can still operate, but an additional factor begins to play an increasingly important role. This factor is a tendency for novel objects to evoke fear reactions which, if they are strong enough, will compete with and thus prevent filial behavior. The model assumes that when an organism is exposed to an appropriate imprinting object before the age when fear of novel objects is strong, the classical 
conditioning process occurs and the object is rendered familiar (i.e., the subject learns its features). This insures that the object will not subsequently function as a novel stimulus at later stages of ontogenetic development when novelty-induced fear reactions would compete with the filial response. Objects which have not been encountered during this early stage would not have this immunity to novelty-induced fear.

These considerations have several implications. First, they imply that the so-called "critical period for imprinting" is that interval early in a subject's life (Days 1 and 2) before it exhibits strong fear reactions to objects that are sources of reinforcing stimulation but which are novel. They also imply that imprinting should take place in older subjects provided that novelty-induced fear reactions can be eliminated. Moreover, if the model is correct, then imprinting to one stimulus at an early age should not necessarily prevent imprinting to a different stimulus at a later age (again assuming that novelty-induced fear reactions can be eliminated). The research reported here was designed to assess these implications of the reinforcement model of imprinting.

\section{EXPERIMENT 1}

The reinforcement model of imprinting implies that older subjects should be capable of forming imprinting attachments. Several experiments have yielded data that are consistent with this proposition, but in all of them the index of imprinting was some measure of either approach behavior or distress calling (see Ratner \& Hoffman, 1974, and Sluckin \& Salzen, 1961, for summaries of these studies).

Experiment 1 sought to extend the investigation of imprinting in older subjects by examining an aspect of behavior that has not been studied in the prior work. Ducklings that have been imprinted to a given stimulus on the first or second day after hatching can be readily trained to perform an operant response in order to view that stimulus (Hoffman, Searle, Toffey, \& Kozma, 1966; Peterson, 1960). Further, these responses tend to occur in bursts, rather than being evenly distributed over time (Hoffman \& Kozma, 1967). The present experiment asked whether ducklings first exposed to an imprinting stimulus at an older age (Day 10 posthatch) could also be trained to perform an operant response when presentation of the imprinting stimulus served as the reinforcing event, and if they could, whether they too would exhibit burst-like response patterns. Such effects would be consistent with the reinforcement model, but they would be inconsistent with the classical view which holds that attachments formed after the critical period are somehow different from those formed during this period (Hess, 1973). As a sub- sidiary issue, this experiment also compared ducklings' responding when the imprinting stimulus was a mechanical object with their responding when the imprinting stimulus was another duckling. Some researchers make a distinction between a bird's attachment to its mother or mother surrogate and its attachment to siblings (e.g., Hess, 1973; Lorenz, 1935). The reinforcement model of imprinting does not draw a sharp distinction between the two kinds of attachment, since it assumes that both reflect the same basic conditioning processes.

\section{Method}

Subjects. Twelve Peking ducklings (Anas platyrhynchos) were hatched from eggs obtained from the $C \&$ R Duck Farm, Inc., Long Island, New York.

Apparatus. The experimental chamber (see Figure 1) consisted of a plywood box $(140 \times 64 \times 76 \mathrm{~cm})$ divided lengthwise by a fine-mesh stainless steel screen into two equal-sized compartments-a stimulus compartment and a subject compartment. Lighting in the subject compartment was provided by two continuously illuminated $75-\mathrm{W}$ incandescent lamps mounted above the screen. These lamps were positioned so that, unless the stimulus compartment was also illuminated, the light reflected from the stainless steel screen prevented the subject from viewing into the stimulus compartment.

Two imprinting stimuli were employed. The stimulus compartment contained only one of these stimuli at any given time. The object shown in Figure 1 was a white foam-rubber object (30 $\times 10 \times 10 \mathrm{~cm}$ ) mounted over a model train engine. Presentations of this stimulus were produced by switching on two overhead 75-W incandescent lamps in this section of the stimulus compartment and moving the stimulus back and forth along HOgauge track at approximately $30 \mathrm{~cm} / \mathrm{sec}$. Stimulus withdrawal was accomplished by switching off the overhead lamps and stopping the movement of the stimulus.

The second imprinting stimulus was a duckling of the same age as the subject tested. This duckling was confined within a smaller enclosure $(25 \times 40 \mathrm{~cm})$ in the center of the stimulus compartment. With this arrangement the duckling in the enclosure could always view the subject, but the subject could only view the duckling in the enclosure when the stimulus compartment was illuminated.

A balsa-wood pole $(1 \times 1 \times 30 \mathrm{~cm})$, which was attached to a pigeon key mounted on the wall above the center of the stainless steel screen, hung vertically down to the floor of the subject compartment. When the pole was operative, a peck with a force of at least $2 \mathrm{~g}$ yielded a stimulus presentation of a predetermined duration.

An Esterline-Angus operations recorder, used to document pole pecks and stimulus events, and standard relay programming equipment were located in an adjacent room. The subject's behavior was monitored via a closed-circuit television system.

Procedure. Approximately $12 \mathrm{~h}$ after hatching, the ducklings were removed from the incubator and placed in individual housing units. Each duckling was kept in visual isolation, but could hear

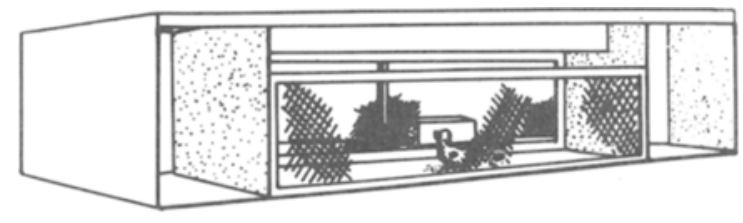

Figure 1. Apparatus used for imprinting and pole-peck training. 
the ducklings in the other housing units. The birds were maintained on a natural day-night cycle and had free access to food and water.

After approximately $8 \mathrm{~h}$ in their housing units, six of the ducklings received their first imprinting session. Each bird was placed in the test apparatus and given $\mathbf{4 5} \mathrm{min}$ of continuous exposure to the imprinting stimulus. For three of the birds, this stimulus was the moving foam rubber object; for the remaining three birds, the stimulus was another duckling. The following day, each bird was given a second 45-min imprinting session.

Training of the pecking response for these early imprinting subjects took place on the third day after hatching. Presentation of the particular stimulus to which a given duckling had been imprinted served as the reinforcing event for that duckling. The experimenter observed the duckling in the apparatus and presented the stimulus for very short durations (approximately $0.5 \mathrm{sec}$ ) when the subject approached the pole. Once the duckling was in the vicinity of the pole, only motions which more and more closely resembled a pole peck were reinforced, until the duckling actually pecked the pole. The first peck produced the stimulus for $5 \mathrm{sec}$ and, as the response became stronger, the duration was gradually increased to $10 \mathrm{sec}$. After training was completed, each duckling was given $2 \mathrm{~h}$ of pecking experience, during which each peck produced the stimulus for $10 \mathrm{sec}$. Ducklings that emitted at least 100 reinforced pecks over the 2 -h session were considered to have met the criterion for successful learning of the pole-peck response. On the day following this 2 -h session (Day 4), the duckling with the highest rate in each stimulus condition was given a 6-h pole-pecking session in which each response produced a 10-sec presentation of the stimulus.

The remaining six ducklings in this experiment were maintained in visual isolation for the first 10 days posthatch. On Day 10, each subject was placed in the test apparatus and received a single imprinting session of $16 \mathrm{~h}$ continuous exposure to the imprinting stimulus. For three of the birds, this stimulus was another duckling, and for the remaining three birds, this stimulus was the foam rubber object. On Day 12 posthatch, each duckling was trained to peck the hole and given $2 \mathrm{~h}$ of pecking experience in the manner previously described. On the day following this session, the duckling with the highest rate in each condition was given a 6 -h pole-pecking session in which each response produced a $10-\mathrm{sec}$ presentation of the stimulus.

\section{Results}

During the imprinting sessions, the six early imprinting birds almost immediately began following the stimulus and maintained close proximity to it. In contrast, the late imprinting birds initially gave distress vocalizations and attempted to escape from the stimulus, whether the stimulus was the foam rubber object or another duckling. But by the end of the exposure period, all of these birds displayed the same approach and following behavior evidenced by the early imprinting subjects.

All 12 birds learned to peck the pole and each emitted at least 100 reinforced pecks during the 2 -h criterion session. Thus, both the foam rubber object and another duckling were effective reinforcers after late imprinting as well as after early imprinting. Figure 2 presents the response records for the four subjects (one at each reinforcement condition and at each age) that were tested in the 6-h pole-peck session. It is evident that both the early imprinting birds and the late imprinting birds appeared to emit bursts of responses, rather than isolated pecks. These
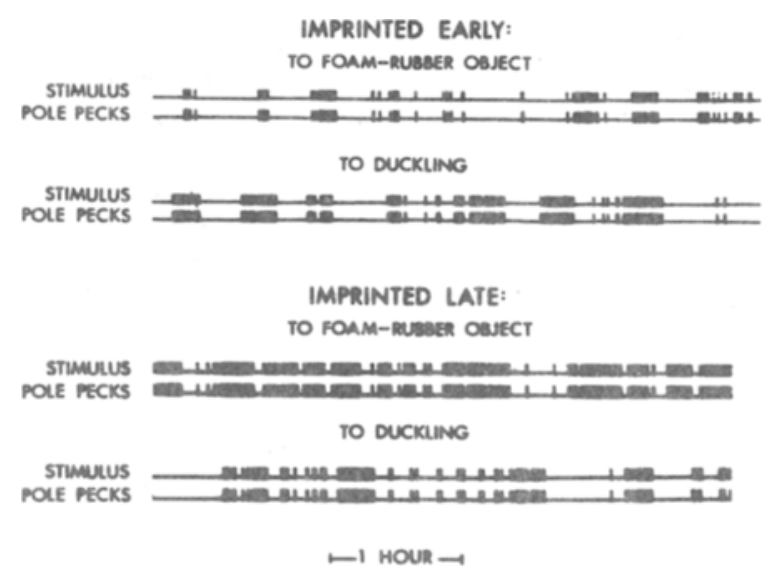

Figure 2. Response records for the four ducklings (one in each experimental condition) given extended pole-peck training.

bursts of responses imply that response emission involved a form of sequential dependency. The reliability of this sort of response pattern can be determined by an analysis of first-order conditional probabilities. Table 1 shows these probabilities for the four ducklings. The response records were divided into small equal time units $(1 \mathrm{~mm}=1$ unit $=$ approximately $2.0 \mathrm{~min}$ ). Across the period studied, there were differences between the four ducklings in the probability that a given time unit contained one (or more) responses $[\mathrm{P}(\mathrm{r})]$. For example, the late-imprinted duckling pecking for the foam rubber object displayed a higher probability of responding than did the other ducklings. More important, however, is the relationship for each of the ducklings between two conditional probabilities. One of these is the probability that a time unit contained a response given that the previous time unit contained a response $[P(r / r)]$. The other is the probability that a time unit contained a response given that the previous time unit contained no response $[\mathrm{P}(\mathrm{r} / \mathrm{r})]$.

Table 1

Conditional Probabilities of Pole-peck Responses For One Duckding From Each of the Four Experimental Conditions.

\begin{tabular}{llll}
\hline & \multicolumn{3}{l}{ Response Probabilities } \\
\cline { 2 - 4 } & $\mathrm{P}(\mathrm{r})$ & $\mathrm{P}(\mathrm{r} / \mathrm{r})$ & $\mathrm{P}(\mathrm{r} / \overline{\mathrm{r}})$ \\
\hline Imprinted Early: & & & \\
$\quad$ - to foam rubber object & .34 & .69 & .16 \\
$\quad$-to duckling & .43 & .81 & .14 \\
Imprinted Late: & & & \\
$\quad$-to foam rubber object & .74 & .84 & .43 \\
$\quad$-to duckling & .46 & .78 & .19 \\
\hline
\end{tabular}

Note- $P(r)=$ the probability that a given 2-min interval contained one or more responses. $P(r / r)=$ the probability that a given interval contained at least one response, given that the pre. ceding interval also contained at least one response. $P(r / 7)=$ the probability that a given interval contained at least one response, given that the preceding interval contained no responses. 
For each duckling, $\mathrm{P}(\mathrm{r} / \mathrm{r})$ was significantly greater than $P(r / r)(z \geqslant 5.46, p<.05)$. Since $P(r / r)$ would be expected to approximate $P(r / r)$ if responses were emitted independently of each other, the finding of significant differences between these two conditional probabilities means that the sequential dependencies evident in the data were reliable for each bird. Thus, whether the ducklings received early or late imprinting, or whether they were imprinted to the foam rubber object or another duckling, they showed a strong tendency to peck for the stimulus in burst-like sequences.

\section{Discussion}

These results indicate that both the foam rubber object and another duckling were effective reinforcers of operant responding in ducklings imprinted early in life (on Day 1) and also in ducklings imprinted later in life (on Day 10). Further, regardless of the age at which the ducklings were imprinted or the particular imprinting stimulus employed, the subjects pecked for the stimulus in burst-like patterns. These data provide no comfort for a theory which holds that imprinting effects are restricted to mother or mother surrogates and do not apply to siblings (Hess, 1973). Nor do these data support the contention of traditional interpretations which hold that imprinting effects are restricted to an early socalled critical period in the subject's life. These data are, however, consistent with the reinforcement model of imprinting.

\section{EXPERIMENT 2}

Previous research has suggested that imprinting to one stimulus does not preclude subsequent imprinting to a second stimulus (Hoffman, Ratner, \& Eiserer, 1972). In that study, the capacity of the second stimulus to suppress ongoing distress vocalizations was used as a measure of imprinting. Experiment 2 sought to extend this finding by determining whether an imprinting stimulus could function as a reinforcer of operant responding in ducklings with a prior history of imprinting to a different stimulus. Such a finding is not predicted by the traditional view of imprinting. Further, the traditional view predicts that, given a choice between the two stimuli, a bird will choose the initially presented stimulus (the primary effect). In contrast, the reinforcement model of imprinting offers no reason why a second stimulus should not be an effective reinforcer, nor does it preclude the possibility that a preference for the second stimulus might develop.

\section{Method}

Subjects. The subjects were 11 naive ducklings.

Apparatus. The apparatus used for the pole-pecking sessions was identical to that employed in the first experiment. For the preference tests, only the subject compartment of the apparatus was employed the stimulus compartment remained in darkness). Two wire-mesh screens were inserted in the subject compartment, dividing it widthwise into three equal-sized compartments. The lights in the apparatus were arranged so that a duckling placed in the middle compartment could see into the two outer compartments. On each preference test, a conspecific was always placed in one outer compartment and the foam rubber object was placed in the other outer compartment. The foam rubber object was suspended by a thread so that it hung approximately $2 \mathrm{~cm}$ above the floor, with its long axis parallel to the screen which separated the middle and outer compartments. Another thread was attached to the end of the object closest to the front wall. This thread ran out of the compartment and was held by an experimenter who, by gently pulling on the thread, could swing the stimulus back and forth, simulating the movement of the stimulus when mounted on the train. Throughout the preference test, a recording of the sound of the train moving back and forth was played on a tape recorder behind the outer compartment. The experimenter quietly sat in front of the apparatus (invisible to the subject) and documented the subject's behavior by means of a push-button control panel attached to an Esterline-Angus operations recorder.

Procedure. Approximately $12 \mathrm{~h}$ after hatching, the ducklings were removed from the incubator and placed in individual housing units. Approximately $8 \mathrm{~h}$ later, each subject was placed in the test apparatus and given $45 \mathrm{~min}$ of continuous exposure to an imprinting stimulus. For six of the birds, this stimulus was another duckling, and for the remaining five birds, the stimulus was the foam rubber object. On the second day after hatching, each duckling was shaped to peck the pole in order to receive presentations of the stimulus to which it had been imprinted. Following shaping, each duckling was given a 1 -h session in which each peck produced the imprinting stimulus for $10 \mathrm{sec}$. On Days 3 and 4 , the subjects were given two further 1 -h pole-peck sessions. Beginning on Day 5, on each successive day one bird from each stimulus condition was switched from pecking for its original imprinting stimulus to pecking for the novel stimulus. If necessary, shaping was used. Thus, by Day 9 , all five ducklings originally trained to peck for the foam rubber object had been switched to pecking for the conspecific (object-to-conspecific condition), and by Day 10, all six ducklings originally trained with the conspecific had been switched to pecking for the foam rubber object (conspecific-to-object condition). After being switched to the new stimulus, each duckling continued pecking for the new stimulus until it had received a total of 12 1-h sessions or until it had changed its preference (see below) from the initial stimulus to the new stimulus. A duckling was considered to have met criterion for successful learning of the pecking response with a given reinforcer if it averaged at least 50 pecks/1-h session across all sessions in which that reinforcer was employed.

Immediately before and after each pecking session with the new stimulus, a 5 -min preference test between the two stimuli was conducted. At the start of each test, the duckling was placed in the center of the middle compartment. A preference for one stimulus was scored if the subject spent at least $75 \%$ of its time on the side of the middle compartment nearest that stimulus and was facing towards that stimulus. Both of these criteria had to be met in order for a preference to be scored. A subject was considered to have changed its preference if it preferred the new (second) imprinting stimulus on four successive tests. The side on which each stimulus was located was randomly varied from test to test for each subject.

\section{Results}

All subjects pecked for the initial imprinting stimulus at an average rate of at least 50 pecks $/ 1$-h session. During the first pole-peck session with the novel stimulus, the initial presentation of that stimulus 
produced a startle response in all ducklings. Following the first peck, two ducklings in the object-toconspecific condition and three ducklings in the conspecific-to-object condition required shaping before they would resume pecking for the new stimulus. The remaining six ducklings (three in each condition) maintained their pecking without shaping. In the subsequent pole-peck sessions, all subjects pecked for the second imprinting stimulus at a rate of at least 50 pecks/session.

On the first preference test, all birds preferred the stimulus to which they had initially been exposed. On the subsequent preference tests, four of the six birds in the conspecific-to-object condition continued to prefer the conspecific despite $12 \mathrm{~h}$ of polepecking for the foam rubber object. The two remaining birds in this condition changed their preference to the foam rubber object after an average of $3 \mathrm{~h}$ of pole-pecking for that stimulus. These two birds were the last two subjects in their condition to be switched from pecking for the conspecific to pecking for the object-on the 9th and 10th days after hatching. All five birds in the object-to-conspecific condition changed their preference to the conspecific. This change in preference occurred after an average of $5.7 \mathrm{~h}$ of pole-pecking for the conspecific.

\section{Discussion}

It is clear that prior imprinting and pole-peck experience with one stimulus does not preclude the ability of a second imprinting stimulus to reinforce operant responding. Further, most of the subjects in the present study came to choose the second stimulus over the first in a preference test. These results are obviously inconsistent with the view that imprinting is an irreversible process which precludes the formation of subsequent attachments. They are, however, entirely consistent with the reinforcement model of imprinting, which assumes that the critical factor for determining whether or not an imprinting stimulus will support operant responding is simply whether the stimulus in question has unconditioned reinforcing properties. Since it is known from Experiment 1 that both the conspecific and the foam rubber object have these properties, the reinforcement model of imprinting predicts that these stimuli should support operant responding regardless of the subject's prior imprinting experience. With respect to the preference test data, the reinforcement model predicts that the subject's preference should depend on the relative strengths of the unconditioned features of the two stimuli and on whether or not the initially neutral features of each stimulus have been conditioned. More specifically, the model predicts that a subject exposed to one imprinting stimulus early in life and a second imprinting stimulus later in life should initially prefer the former stimulus since that stimulus is familiar, and hence not fear eliciting. After the second stimulus becomes familiar through classical conditioning, however, the subject's preference for a given stimulus should be determined by which stimulus has the more powerful unconditioned properties. If one makes the intuitively reasonable, though admittedly post hoc, assumption that a conspecific has, in general, stronger unconditioned properties than a foam rubber object, the model is consistent not only with the fact that some subjects change their preference, but also with the fact that all of the ducklings in the object-to-conspecific condition, but only two of the six ducklings in the conspecific-to-object condition, changed their preferences.

It should be noted that the two ducklings in the conspecific-to-object condition that came to prefer the object were the subjects that had the most exposure to the conspecific in this group. This argues against the possibility that these subjects changed their preference simply because they did not have sufficient time to become well imprinted to the conspecific.

One aspect of the pecking data deserves further comment. All subjects pecked for the second stimulus despite the fact that it was novel and hence fear eliciting. Several factors may account for why this happened. Prior research has shown that the presentation of a novel, fear-eliciting object enhances polepecking reinforced by a stimulus to which a duckling has already been imprinted (Hoffman et al., 1966). Thus, a duckling in the present study, when confronted with the novel stimulus, might be expected to continue performing a response which had previously produced the original imprinting stimulus. The consequence of these pecks, however, was to bring the subject into repeated contact with the novel stimulus. Ratner and Hoffman (1974) found that repeated exposure to a novel imprinting stimulus is an effective procedure for eliminating the fear reactions that such stimuli elicit in older ducklings. They also found that once these fear reactions had been reduced, rather than responding with indifference, the ducklings reacted filially. This implied that the fear reactions had competed with, and in this fashion precluded, an innate tendency to react filially to the reinforcing aspects of the stimulus. Their findings are consistent with the present finding that all ducklings persisted in pecking for the novel stimulus and that some even came to prefer it.

\section{EXPERIMENT 3}

Experiment 3 examined another possible parallel between the imprinting reactions of naive, newly hatched ducklings and those of older, previously imprinted ducklings. Hoffman, Eiserer, and Singer 
(1972) found that while the initial presentation of a moving mechanical object to newly hatched ducklings completely suppressed ongoing distress calling, presentation of the object stationary did not suppress calling until the ducklings had seen the object in motion for some time. In terms of the classical conditioning model this implied that the stimulation provided by visual movement could innately evoke filial reactions and that while the static features of the object (e.g., its color, shape, and size) were initially neutral, they could serve as conditioned elicitors of filial behavior after the object had been presented in motion. Eiserer, Hoffman, and Klein (1975) further found that once the static features of the object acquired the capacity to suppress ongoing distress calling, they maintained that capacity for many sessions-even though the object was never again seen in motion. The present study asked whether similar effects would be obtained in ducklings with a prior history of imprinting to a different object.

\section{Method}

Subjects. The subjects were four naive ducklings.

Apparatus. The experimental chamber was identical to that described in Experiment 1. Two imprinting objects were employed. One was the foam rubber object mounted over the model train, as previously described; the other was rotating lamp of the sort used on the top of many police vehicles and ambulances (see Hoffman et al., 1972, for details).

The remaining equipment was identical to that used in Experiment 1 except that a specially constructed voice key that was sensitive only to sounds in the frequency range of the distress call $(3,000-4,000 \mathrm{~Hz})$ was used to detect distress vocalizations.

Procedure. At $12-16 \mathrm{~h}$ posthatch, the ducklings were transferred from the incubator to the subject compartment of the imprinting apparatus. Here each duckling was individually exposed to the rotating lamp for $30 \mathrm{~min}$. The subjects received four additional 30-min imprinting sessions with the rotating lamp over the next 2 days.

Immediately following the last imprinting session, each duckling was given a test in order to assess the ability of the rotating lamp to suppress distress calls. This test consisted of three 12 sec presentations of the rotating lamp, each of which was preceded by a 12 -sec period during which the lamp was not visible.

On Day 5 posthatch, each duckling was given a second distress vocalization test, but this time the stimulus consisted of the foam rubber object (the lamp having been removed from the apparatus). The test was conducted identically to the test previously described, except that three sets of 12 -sec presentations and withdrawals of the stationary object were given subsequent to the three sets of presentations and withdrawals of the moving object.

Following this test and extending through Day 6, each duckling received five 30 -min imprinting sessions with the moving foam rubber stimulus constantly visible. Subsequent to each imprinting session, a distress vocalization test identical to the second test was administered.

On Days 7-16 posthatch, each duckling received $1030-\mathrm{min}$ sessions during which the stationary foam rubber object was presented continuously. Each session was followed by a distressvocalization-suppression test identical in procedure to the tests given previously, except that the presentations of the moving stimulus were omitted. Thus, after Day 6, the ducklings never saw the object in motion.

\section{Results}

For a given subject on a suppression test, suppression of distress vocalizations by a given stimulus was assessed by calculating the ratio: (B-S)/ $(B+S)$, where $B$ represents seconds of distress vocalizations during the 12 -sec stimulus withdrawal period that preceded a given stimulus presentation and $S$ represents seconds of distress vocalizations during the $12-\mathrm{sec}$ presentation of the stimulus. With this index, a ratio of +1.00 indicates perfect suppression by the stimulus, while ratios that approximate zero indicate that distress vocalization in the presence of the stimulus was essentially the same as distress vocalization in its absence.

Figure 3 shows the mean suppression ratios for the rotating lamp during Test 1 , and it also shows the mean suppression ratios for both the moving and stationary foam rubber object during Tests 2-7 and for the stationary foam rubber object only during Tests 8-17. As indicated in Figure 3, presentation of the rotating lamp completely suppressed distress vocalizations on Test 1 , but neither the stationary nor the moving foam rubber object exerted any control over distress vocalizations when first presented on Test 2. After two imprinting sessions, however, both the stationary and the moving foam rubber object exhibited nearly complete suppressive control over distress vocalization (Tests 4-7). There was no appreciable decrease in the amount of suppression produced by the stationary stimulus over Tests 8-17.

Observation via closed-circuit TV revealed that when the moving foam rubber object was first presented during Test 2 , every duckling immediately ran away from it, but with continued exposure, efforts to escape gradually subsided. In the later sessions, the ducklings oriented towards and sometimes followed the object as it traversed the chamber. During Tests 8-17, when the object no longer moved, the duckling usually sat quietly in front of the screen where the object was visible; they never appeared agitated and did not emit distress calls except at the

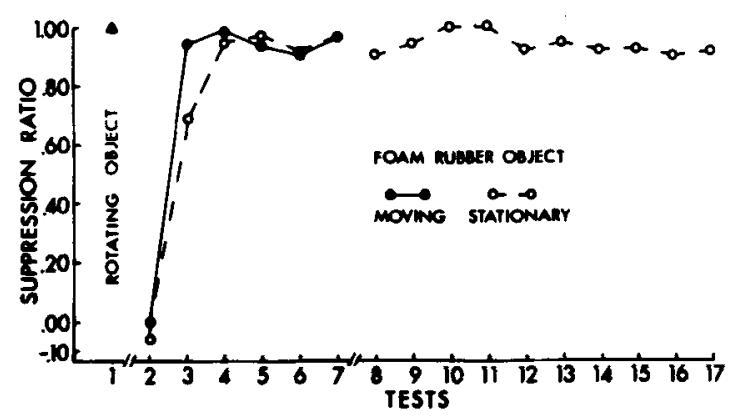

Figure 3. Mean suppression of disiress calling to the rotating lamp and the moving and stationary foam rubber object as a function of test sessions. 
end of each session when the object was briefly withdrawn for the purpose of the distress vocalization tests.

\section{Discussion}

Prior work (Eiserer et al., 1975) has revealed that when ducklings with no prior history of imprinting are exposed to a moving object within the first day of hatching, the static visual features of the object acquire the capacity to suppress distress vocalization, and this capacity persists at maximal strength through many hours of exposure to the static features alone. The present findings show that the static features of such an object acquire these same persistent capacities in older ducklings with a prior nistory of imprinting to a different object. Thus, as in Experiments 1 and 2, we observed a close parallel between the imprinting behavior of older and younger ducklings.

There is, however, an important difference between the present results and the results obtained with younger ducklings. In the present study, the unfamiliar moving object appeared to gradually "acquire" the capacity to suppress distress calls rather than immediately being able to do so. This effect is an expected consequence of the fact that, as previously described, an older duckling's fear of novelty must be reduced before the innately reinforcing properties of a novel imprinting stimulus can be observed. Thus, the gradual increase in control of distress vocalizations by the moving object reflects the gradual habituation of the competing fear response. However, the acquisition function for the stationary object must reflect not only the habituation of fear but also the conditioning of filial reactions to the static features as a result of their association with visual movement. Prolonged exposure of only the static features of such an object to ducklings that are never exposed to the object in motion does not result in the acquisition of suppressive properties (Eiserer \& Hoffman, 1974; Hoffman et al., 1972).

\section{CONCLUSIONS}

Prior investigations have revealed that precocial birds imprinted at an older age come to approach and follow the imprinting stimulus in a manner similar to that exhibited by subjects imprinted during the so-called "critical period" (Ratner \& Hoffman, 1974; Sluckin \& Salzen, 1961). The present research has extended these findings by showing that the reinforcing properties of an imprinting object and its control over distress vocalization are essentially the same whether imprinting has occurred early or late, and by showing that a prior history of imprinting does not prevent a second imprinting object from exhibiting these capacities. In addition, the present research has revealed that when subjects are sequentially imprinted to two stimuli, the order of imprinting is not a critical determinant of the subject's ultimate preference. These findings support a view of imprinting which is far less constrained by factors of age and previous experience than is suggested by the traditional view of imprinting as an irreversible process confined to a brief critical period early in an animal's life. In fact, the only obvious difference we have observed between ducklings imprinted early and ducklings imprinted late is that younger naive ducklings immediately react filially to the presence of a novel imprinting stimulus, while older ducklings initially react fearfully to such a stimulus and only gradually come to react filially. Once the fearful reactions have subsided, however, the entire spectrum of filial reactions is evoked by the imprinting object-approach, following, reinforcement of operant responding, burst-like patterns of operant responding, and suppression of distress calling by both innately reinforcing and initially neutral features of the object. Thus, we conclude that imprinting in older ducklings is a process identical to imprinting in younger ducklings, although, because of competing fear reactions, it takes longer. In all respects, the results of the present research are consonant with the reinforcement model of imprinting.

\section{REFERENCES}

Eibl-Eibesfeldt, I. Ethology: The biology of behavior (2nd ed.). New York: Holt, Rinehart, \& Winston, 1975.

Eiserer, L. A., \& Hoffman, H. S. Acquisition of behavioral control by the auditory features of an imprinting object. Animal Learning \& Behavior, 1974, 2, 275-277.

Eiserer, L. A., Hoffman, H. S., \& Klein, S. H. Persistence of acquired behavioral control in the context of imprinting. Journal of the Experimental Analysis of Behavior, 1975, 24, 255-266.

Hess, E. H. Imprinting. Science, 1959, 130, 133-141.

HESs, E. H. Imprinting: Early experience and the developmental psychobiology of attachment. New York: Van Nostrand Reinhold, 1973.

Hoffman, H. S., Eiserer, L. A., \& Singer, D. Acquisition of behavioral control by a stationary imprinting stimulus. Psychonomic Science, 1972, 26, 146-148.

Hoffman, H. S., \& Kozma, F., JR. Behavioral control by an imprinted stimulus: Long-term effects. Journal of the Experimental Analysis of Behavior, 1967, 10, 495-501.

Hoffman, H. S., \& Ratner, A. M. A reinforcement model of imprinting: Implications for socialization in monkeys and men. Psychological Review, 1973, 80, 527-544.

Hoffman, H. S., Ratner, A. M., \& Eiserer, L. A. Role of visual imprinting in the emergence of specific filial attachments in ducklings. Journal of Experimental Psychology, 1972, 81, 399.401.

Hoffman, H. S., Searle, J. L., Toffey, S., \& Kozma, F., JR. Behavioral control by an imprinted stimulus. Journal of the Experimental Analysis of Behavior, 1966, 9, 177-189.

LORENZ, K. Z. [Companions as factors in the bird's environment.] In K. Lorenz, Studies in animal and human behavior (Vol. 1). Cambridge, Mass: Harvard University Press, 1970. (R. Martin 
trans.) (From Journal für Ornithologie, 1935, 83, 137-213, 289.413.)

Peterson. N. Control of behavior by presentation of an imprinting stimulus. Science, 1960, 132. 1395-1396.

Ratner, A. M.. \& Hoffman, H. S. Evidence for a critical period for imprinting in Khaki Campbell ducklings (Anas platyrhynchos domesticus). Animal Behaviour, 1974, 22, 249-255.
(Received for publication May 13, 1977; accepted August 8, 1977.)
Stuckin, W.. \& SAlzen, E. A. Imprinting and perceptual learning. Quarterly Journal of Experimental Psychology. 1961, 13. $65-77$. 